\title{
SPECTRUM OF THE LAPLACIAN ON MANIFOLDS WITH SPIN(9) HOLONOMY
}

\author{
KWAN-HANG LAM
}

\begin{abstract}
We consider noncompact complete manifolds with Spin(9) holonomy and proved an one end result and a splitting type theorem under different conditions on the bottom of the spectrum. We proved that any harmonic functions with finite Dirichlet integral must be Cayley-harmonic, which allowed us to conclude an one end result. In the second part, we established a splitting type theorem by utilizing the Busemann function.
\end{abstract}

\section{Introduction}

In [13], the authors proved the following

Theorem. [13] Let $M$ be a complete Riemannian manifold with a parallel $p$-form $\omega$. Assume that $f$ is a harmonic function satisfying

$$
\int_{B_{p}(R)}|\nabla f|^{2}=o\left(R^{2}\right)
$$

as $R \rightarrow \infty$, then $f$ satisfies

$$
d *(d f \wedge \omega)=0 .
$$

Combining the above theorem with the fact that a quaternionic Kähler manifold supports a global parallel 4 -form $\omega$, the authors proved, by an explicit calculation involving $\omega$, that a harmonic function with bounded Dirichlet integral is quaternionicharmonic. Utilizing the quaternionic-harmonic condition they proved that, under an assumption on the bottom of the spectrum $\lambda_{1}(M)$, such a manifold must have exactly one infinite volume end. Since a manifold with holonomy group Spin(9) supports a global parallel 8 -form $\Omega$, by a careful and detail study of $\Omega$, we proved that any harmonic functions with bounded Dirichlet integral is Cayley-harmonic. Similar to the work in [13], with a suitable lower bound assumption on $\lambda_{1}(M)$, an one infinite volume end result has been established by utilizing the Cayley-harmonicity condition. In the second part of this paper, we consider the case that $\lambda_{1}(M)=121$ achieves its maximal value. By studying the Busemann function $\beta$ on $M$ and using the results in [10] and [12], we proved that either $M$ has only one end or $M$ must splits as $\mathbb{R} \times N$, where $N$ is given by a level set of $\beta$.

Received by the editors November 19, 2007.

Research was partially supported by NSF grant \#0503735 and NSC grant 96-2115-M-006-017 of the ROC. 


\section{Cayley hyperbolic space}

We give a brief introduction on the Cayley numbers $\mathbb{O}$, and a description of the sectional curvature of the Cayley hyperbolic space $\mathbb{H}_{\mathbb{O}}^{2}$. The material presented here is adopted from [3], we refer the readers to there for further details. The Cayley numbers $(\mathbb{O}$, is an 8-dimensional non-associative division algebra over the real numbers which satisfies the alternative law: $x(x y)=x^{2} y,(y x) x=y x^{2}$. It has a multiplicative identity 1 and a positive definite bilinear form $\langle$,$\rangle whose associated norm \|\cdot\|$ satisfies $\|a b\|=\|a\| \cdot\|b\|$. Every element $a \in \mathbb{O}$ can be written as $a=\alpha 1+a_{0}$, where $\alpha$ is real and $\left\langle a_{0}, 1\right\rangle=0$. The conjugation map $a \mapsto a^{*}=\alpha 1-a_{0}$ is an anti-automorphism, that is $(a b)^{*}=b^{*} a^{*}$. Moreover, $a a^{*}=\langle a, a\rangle 1$ and $\langle a, b\rangle=\left\langle a^{*}, b^{*}\right\rangle$. $\mathbb{O}$ admits a canonical basis $\left\{1, e_{0}, \cdots, e_{6}\right\}$ such that $\left\langle e_{i}, e_{j}\right\rangle=\delta_{i j}, e_{i}^{2}=-1, e_{i} e_{j}+e_{j} e_{i}=0$ for $i \neq j$, and $e_{i} e_{i+1}=e_{i+3}$, if $i$ is an integer mod 7. Obviously, we can extend the positive bilinear form from $\mathbb{O}$ to $\mathbb{O}^{2}$ by

$$
\langle(a, b),(c, d)\rangle=\langle a, c\rangle+\langle b, d\rangle,
$$

where $a, b, c, d \in \mathbb{O}$. For any point $x \in \mathbb{H}_{\mathbb{O}}^{2}$, we make the following identification $T_{x} \mathbb{H}_{\mathbb{O}}^{2} \simeq \mathbb{O}^{2}$. Let $M$ be a Riemannian manifold with metric tensor $\langle$,$\rangle . Let V$ be any tangent space to $M$. The curvature operator of $M$ at $V$ is a map

$$
R: \Lambda^{2}(V) \rightarrow \Lambda^{2}(V) \subseteq \operatorname{Hom}(V, V)
$$

such that

$$
R(x \wedge y) z+R(z \wedge x) y+R(y \wedge z) x=0 .
$$

The above two properties implies $R$ is a symmetric linear operator, that is

$$
\langle R(x \wedge y) z, w\rangle=\langle R(x \wedge y), z \wedge w\rangle=\langle R(z \wedge w), x \wedge y\rangle
$$

for any $x, y, z, w \in V$. For any $x, y \in V$ linearly independent, the sectional curvature of the 2-plane spanned by $x$ and $y$ is defined by

$$
K_{x \wedge y}=\frac{\langle R(x \wedge y), x \wedge y\rangle}{\|x \wedge y\|^{2}} .
$$

The sectional curvature $K_{(a, b) \wedge(c, d)}$ of the 2-plane $(a, b) \wedge(c, d)$ of $\mathbb{O}^{2}$ has the following properties:

(1) For any $a, b, c, d \in \mathbb{O}$ with $\|(a, b)\|=\|(c, d)\|=1$ and $\langle(a, b),(c, d)\rangle=0$, we have

$$
\begin{aligned}
K_{(a, b) \wedge(c, d)} & =\alpha\left\{\|a \wedge c\|^{2}+\|b \wedge d\|^{2}+\frac{1}{4}\|a\|^{2}\|d\|^{2}+\frac{1}{4}\|b\|^{2}\|c\|^{2}\right. \\
& \left.+\frac{1}{2}\langle a b, c d\rangle-\langle a d, c b\rangle\right\}
\end{aligned}
$$

$$
K_{(a, 0) \wedge(b, 0)}=\alpha \text { if }(a, 0) \wedge(b, 0) \neq 0 .
$$

$$
K_{(a, 0) \wedge(0, b)}=\frac{\alpha}{4} \text { if }(a, 0) \wedge(0, b) \neq 0 .
$$

$$
\frac{|\alpha|}{4} \leq\left|K_{(a, b) \wedge(c, d)}\right| \leq|\alpha| \text { if }(a, b) \wedge(c, d) \neq 0 .
$$


In this article, we use the normalization that $\alpha=-4$, hence the sectional curvature of $\mathbb{H}_{\mathbb{O}}^{2}$ is pinched between -4 and -1 . Let $M$ be a complete noncompact Riemannian manifold with holonomy group Spin(9). It was proved in [3] that a manifold with holonomy group Spin(9) must be locally symmetric and its universal covering is either the Cayley projective plane or the Cayley hyperbolic space $\mathbb{H}_{\mathbb{D}}^{2}$. Since we are considering noncompact manifolds, its universal covering is $\mathbb{H}_{\mathbb{O}}^{2}$. We first compute the Laplacian of the distance function of $\mathbb{H}_{\mathbb{D}}^{2}$.

Proposition 1. Let $r(x)=r_{p}(x)$ be the distance function of $\mathbb{H}_{\mathbb{O}}^{2}$ from a fixed point $p$, then

$$
\triangle r=14 \operatorname{coth} 2 r+8 \operatorname{coth} r .
$$

Proof. Let $\gamma:[0, L] \rightarrow M$ be a normal geodesic from $p$ to $x$. Let $e_{1}(t)=\gamma^{\prime}(t)$ along $\gamma$. Let $\left\{e_{A}\right\}_{A=2}^{16}$ be a basis of $T_{p} \mathbb{H}_{\mathbb{O}}^{2}$ such that

$$
\left\{\begin{array}{c}
R_{1 i 1 i}=-4, \quad 2 \leq i \leq 8 \\
R_{1 \alpha 1 \alpha}=-1, \quad 9 \leq \alpha \leq 16 .
\end{array}\right.
$$

We extend $\left\{e_{A}\right\}$ to be a local frame along $\gamma(t),\left\{\gamma^{\prime}(t)=e_{1}(t), e_{2}(t), \cdots, e_{16}(t)\right\}$ by parallel transporting along $\gamma$. Since $\mathbb{H}_{\mathbb{O}}^{2}$ is a symmetric space and thus locally symmetric, we have

$$
\frac{\partial}{\partial t} R_{1 A 1 A}=R_{1 A 1 A, 1}=0,2 \leq A \leq 16,
$$

hence (1) is valid along $\gamma$. Let $X_{A}(t)=f_{A}(t) e_{A}(t)$ be the Jacobi field along $\gamma$ with $X_{A}(0)=0, X_{A}(L)=e_{A}(L) \cdot f_{A}(t)$ satisfies the Jacobi equation

$$
\begin{aligned}
& \frac{d^{2}}{d t^{2}} f_{A}(t)-c_{A}^{2} f_{A}(t)=0 \\
& f_{A}(0)=0, f_{A}(p)=1, \quad 2 \leq A \leq 16 .
\end{aligned}
$$

where $c_{i}=2,2 \leq i \leq 8$ and $c_{\alpha}=1,9 \leq \alpha \leq 16$. Solving the above equation, we have

$$
f_{A}(t)=\frac{\sinh \left(c_{A} t\right)}{\sinh \left(c_{A} L\right)}, 2 \leq A \leq 16 .
$$

Now, we can compute the Hessian of $r$ at $x$

$$
\begin{aligned}
H(r)\left(e_{A}, e_{A}\right) & =\int_{0}^{L}\left(\left|\frac{d X_{A}}{d t}\right|^{2}-\left\langle R\left(X_{A}, \gamma^{\prime}\right) \gamma^{\prime}, X_{A}\right\rangle\right) d t \\
& =\int_{0}^{L}\left(\left|\frac{d f_{A}}{d t}\right|^{2}+c_{A}^{2} f^{2}\right) d t \\
& =c_{A} \operatorname{coth}\left(c_{A} L\right) .
\end{aligned}
$$

Therefore, we conclude that

$$
\begin{aligned}
\triangle r & =\sum_{A=2}^{16} H(r)\left(e_{A}, e_{A}\right) \\
& =14 \operatorname{coth} 2 r+8 \operatorname{coth} r,
\end{aligned}
$$

where we have used the fact that $H(r)\left(e_{1}, e_{1}\right)=0$. 
Theorem 2. Let $M$ be a locally symmetric space with universal covering $\mathbb{H}_{\mathbb{D}}^{2}$. Then

$$
\lambda_{1}(M) \leq 121,
$$

and

$$
\triangle_{M} r \leq 14 \operatorname{coth} 2 r+8 \operatorname{coth} r
$$

in the sense of distribution.

Proof. Let $A(r), V(r)$ be the area and volume of the geodesic ball of radius $r$ of $\mathbb{H}_{\mathbb{O}}^{2}$ respectively. By proposition 1, we have

$$
\frac{A^{\prime}(r)}{A(r)}=14 \operatorname{coth} 2 r+8 \operatorname{coth} r
$$

hence

$$
\begin{aligned}
V_{M}(p, r) & \leq V(r) \\
& =\int_{0}^{r} A(t) d t \\
& \leq C \int_{0}^{r}(\sinh 2 t)^{7}(\sinh t)^{8} d t \\
& \leq C_{1} e^{22 r},
\end{aligned}
$$

where $V_{M}(p, r)$ is volume of the geodesic ball with radius $r$ centered at $p$ and for some constant $C_{1}$. On the other hand, it was shown in [10] that

$$
V_{M}(p, r) \geq C_{2} \exp \left(2 \sqrt{\lambda_{1}(M)} r\right)
$$

for any manifolds with positive spectrum. Combining the above inequality with (3), we conclude that $\lambda_{1}(M) \leq 121$. For the second part, let $f(r)=14 \operatorname{coth} 2 r+8 \operatorname{coth} r$. By proposition 1, we have

$$
\triangle_{M} r(x)=f(r(x))
$$

for any $x \in M \backslash \operatorname{Cut}(p)$, where $\operatorname{Cut}(p)$ is the cut locus of $p$. For each direction $\theta \in S_{p}(M)$, let $R(\theta)=\sup _{t>0}\left\{t: r_{p}\left(\exp _{p}(t \theta)\right)=t\right\}$. Let $\phi \in C_{0}^{\infty}(M)$ be a nonnegative smooth function with compact support, then

$$
\begin{aligned}
\int_{M} \phi f(r) & =\int_{S_{p}(M)} \int_{0}^{R(\theta)} \phi f(r) J(\theta, r) d r d \theta \\
& =\int_{S_{p}(M)} \int_{0}^{R(\theta)} \phi \frac{\partial J}{\partial r} d r d \theta \\
& =-\int_{M} \frac{\partial \phi}{\partial r}+\int_{S_{p}(M)} \phi(\theta, R(\theta)) J(\theta, R(\theta)) d \theta \\
& \geq-\int_{M}\langle\nabla \phi, \nabla r\rangle \\
& =\int_{M} r \triangle \phi,
\end{aligned}
$$

where the second equality follows from the fact that $\triangle r=\frac{\partial}{\partial r}(\log J)$, for all $r<R(\theta)$ and the third equality follows from integration by parts, $\phi \geq 0$ and $J(\theta, 0)=0$. Hence the second result follows. 
Let us recall the definition of the Busemann function and some of its properties. Let $M$ be a complete manifold and $\gamma:[0,+\infty) \rightarrow M$ be a geodesic ray. Let $\beta_{\gamma}^{t}(x)=$ $t-r(\gamma(t), x)$, where $r(x, y)$ denotes the distance between $x$ and $y$. Triangle inequality implies

$$
\left|\beta_{\gamma}^{t}(x)\right|=|r(\gamma(t), \gamma(0))-r(\gamma(t), x)| \leq r(\gamma(0), x)
$$

and

$$
\beta_{\gamma}^{t}(x)-\beta_{\gamma}^{s}(x)=t-s+r(\gamma(s), x)-r(\gamma(t), x) \geq 0,
$$

if $t>s$. Hence $\left\{\beta_{\gamma}^{t}\right\}_{t \geq 0}$ is uniformly bounded on compact subsets of $M$ and nondecreasing, it converges uniformly on any compact subsets of $M$. The Busemann function with respect to a geodesic ray $\gamma$ is defined as

$$
\beta(x)=\lim _{t \rightarrow+\infty} \beta_{\gamma}^{t}(x) .
$$

The following lemma is well-known, see [9] for instance.

\section{Lemma 3.}

almost everywhere.

$$
|\nabla \beta|=1
$$

\section{Manifolds with parallel forms}

Let us first recall the Hodge star operator ${ }^{*}$ and some of its basic properties. Let $V^{n}$ be a $n$-dimensional oriented real inner product space, we have the Hodge star operator

$$
*: \wedge^{p} V \rightarrow \wedge^{n-p} V
$$

for any $\theta \in \wedge^{1} V, v \in V$, exterior multiplication and interior product operators

$$
\begin{aligned}
\varepsilon(\theta): & & \wedge^{p} V \rightarrow \wedge^{p+1} V \\
l(v): & & \wedge^{p} V \rightarrow \wedge^{p-1} V,
\end{aligned}
$$

where $\varepsilon(\theta) \omega=\theta \wedge \omega$ and $(l(v) \omega)(\cdot)=\omega(v, \cdot)$ for any $\omega \in \wedge^{p} V$. Let $\theta, \theta^{\prime} \in \wedge^{1} V$ and $v, v^{\prime} \in V$ be the dual of $\theta$ and $\theta^{\prime}$ respectively with respect the inner product of $V$. For any $\eta \in \wedge^{p} V$, we have the following basic properties

(1) $* * \eta=(-1)^{p(n-p)} \eta$

(2) $* \varepsilon(\theta) \eta=(-1)^{p} l(v) * \eta$

(3) $\varepsilon(\theta) * \eta=(-1)^{p-1} * l(v) \eta$

(4) $* \varepsilon(\theta) * \eta=(-1)^{(p-1)(n-p)} l(v) \eta$

(5) $l(v) \varepsilon\left(\theta^{\prime}\right) \eta+\varepsilon(\theta) l\left(v^{\prime}\right) \eta=0$, where $v \perp v^{\prime}$

(6) $l(v) \varepsilon(\theta) \eta+\varepsilon(\theta) l(v) \eta=\eta$

The following theorem is an over-determined system of equations satisfied by harmonic functions and generalized Corlette's argument to harmonic functions with finite Dirichlet integral on a complete manifold with a parallel $p$-form. This kind of result was first proved by Siu [14] for harmonic maps in his proof of the rigidity theorem for Kähler manifolds. Corlette [5] gave a more systematic approach for harmonic maps with finite energy from a finite volume quaternionic hyperbolic space or Cayley hyperbolic plane to a manifold with nonpositive curvature. In [7], the author generalized Siu's argument to harmonic functions with finite Dirichlet integral on Kähler manifolds. 
Theorem 4. ([13]) Let $M$ be a complete Riemannian manifold with a parallel $p$-form $\omega$. Assume that $f$ is a harmonic function satisfying

$$
\int_{B_{p}(R)}|\nabla f|^{2}=o\left(R^{2}\right)
$$

as $R \rightarrow \infty$, then $f$ satisfies

$$
d *(d f \wedge \omega)=0 .
$$

By taking a careful and closer look at the nature of the proof of the above theorem, we found out that the proof not only works for harmonic functions with finite Dirichlet integral but also $L^{2}$ harmonic 1-form. The key ingredient is that any $L^{2}$ harmonic 1-form is both closed and co-closed. We have the following:

Theorem 5. Let $M^{n}$ be a $n$ dimensional complete Riemannian manifold with a parallel $p$-form $\omega$. Assume that $\alpha$ is a $L^{2}$ harmonic 1-form, that is $\triangle \alpha=0$ and

$$
\int_{M}|\alpha|^{2}<+\infty
$$

Then $\alpha$ satisfies

$$
d *(\alpha \wedge \omega)=0
$$

Proof. We first show that

$$
* d *(\alpha \wedge \omega)=(-1)^{n-1} d *(\alpha \wedge * \omega) .
$$

For any $x \in M$, we choose a local orthonormal frame $\left\{e_{i}\right\}_{i=1}^{n}$ such that $\nabla_{e_{i}} e_{j}(x)=0$. Let $\left\{\theta^{i}\right\}_{i=1}^{n}$ be the coframe. For any $p$-form $\omega$, we have

$$
d \omega=\varepsilon\left(\theta^{i}\right) \nabla_{e_{i}} \omega
$$

at $x$ and $\omega$ is parallel if and only if $\nabla_{e_{i}} \omega=0, \forall i$. Let $\alpha=\sum_{i=1}^{n} a_{i} \theta^{i}$, and hence $\bar{\alpha}=$ $\sum_{i=1}^{n} a_{i} e_{i}$ is the dual of $\alpha$. We use the notation $d \alpha=\sum_{i, j=1}^{n} a_{i, j} \theta^{j} \wedge \theta^{i}$, where $a_{i, j}=$ $\nabla_{e_{j}} a_{i}$. Since $\alpha$ is $L^{2}$ harmonic, it is both closed and co-closed, which are equivalent to the conditions that $a_{i, j}=a_{j, i}$ and $\sum_{i=1}^{n} a_{i, i}=0$. The following calculations are all evaluated at $x$.

$$
\begin{aligned}
d *(\alpha \wedge * \omega) & =d * \varepsilon(\alpha) * \omega \\
& =(-1)^{(p-1)(n-p)} d[l(\bar{\alpha}) \omega] \\
& =(-1)^{(p-1)(n-p)} \sum_{i=1}^{n} \varepsilon\left(\theta^{i}\right) \nabla_{e_{i}}(l(\bar{\alpha}) \omega) \\
& =(-1)^{(p-1)(n-p)} \sum_{i, j=1}^{n} \varepsilon\left(\theta^{i}\right) a_{j, i}\left(l\left(e_{j}\right) \omega\right)
\end{aligned}
$$


where the third equality follows from $\nabla_{e_{i}} e_{j}(x)=0$ and the last equality follows from $\nabla \omega=0$. On the other hand,

$$
\begin{aligned}
(6) * d *(\alpha \wedge \omega)= & * d * \varepsilon(\alpha) \omega \\
= & * \sum_{i=1}^{n} \varepsilon\left(\theta^{i}\right) \nabla_{e_{i}}\left(* \varepsilon\left(\sum_{j=1}^{n} a_{j} \theta^{j}\right) \omega\right) \\
= & *\left(\sum_{i, j=1}^{n} a_{j, i} \varepsilon\left(\theta^{i}\right) *\left(\varepsilon\left(\theta^{j}\right) \omega\right)\right) \\
= & (-1)^{p(n-p-1)} \sum_{i, j=1}^{n} a_{i, j} l\left(e_{i}\right) \varepsilon\left(\theta^{j}\right) \omega \\
= & (-1)^{p(n-p-1)}\left(\sum_{i=1}^{n} a_{i, i} l\left(e_{i}\right) \varepsilon\left(\theta^{i}\right) \omega+\sum_{i \neq j}^{n} a_{i, j} l\left(e_{i}\right) \varepsilon\left(\theta^{j}\right) \omega\right) \\
= & (-1)^{p(n-p-1)}\left(\sum_{i=1}^{n} a_{i, i}\left[\omega-\varepsilon\left(\theta^{i}\right) l\left(e_{i}\right) \omega\right]\right. \\
& \left.-\sum_{i \neq j}^{n} a_{i, j} \varepsilon\left(\theta^{j}\right) l\left(e_{i}\right) \omega\right) \\
= & (-1)^{p(n-p-1)+1} \sum_{i, j=1}^{n} a_{i, j} \varepsilon\left(\theta^{i}\right)\left(l\left(e_{j}\right) \omega\right),
\end{aligned}
$$

where the last equality follows from $a_{i, j}=a_{j, i}$ and $\sum_{i=1}^{n} a_{i, i}=0$. (4) now follows from (5) and (6). Let

$$
\phi(x)=\left\{\begin{array}{ccc}
1 & \text { on } & B_{p}(R) \\
0 & \text { on } & M \backslash B_{p}(2 R)
\end{array}\right.
$$

such that $|\nabla \phi| \leq C_{1} R^{-1}$. Consider

$$
\begin{aligned}
\int_{M} \phi^{2}|d *(\alpha \wedge \omega)|^{2} & =\left|\int_{M} \phi^{2} d *(\alpha \wedge \omega) \wedge * d *(\alpha \wedge \omega)\right| \\
& =\left|\int_{M} \phi^{2} d *(\alpha \wedge \omega) \wedge d *(\alpha \wedge * \omega)\right| \\
& =\left|\int_{M} d \phi^{2} \wedge *(\alpha \wedge \omega) \wedge d *(\alpha \wedge * \omega)\right| \\
& \leq 2\left(\int_{M}|d \phi|^{2}|*(\alpha \wedge \omega)|\right)^{1 / 2}\left(\int_{M} \phi^{2}|d *(\alpha \wedge * \omega)|^{2}\right)^{1 / 2} \\
& =2\left(\int_{M}|d \phi|^{2}|*(\alpha \wedge \omega)|\right)^{1 / 2}\left(\int_{M} \phi^{2}|d *(\alpha \wedge \omega)|^{2}\right)^{1 / 2}
\end{aligned}
$$

where the second and the last equality follows from (4), the third equality follows from integration by parts and the fact that $d^{2}=0 . \omega$ is parallel implies

$$
|*(\alpha \wedge \omega)| \leq C_{2}|\alpha|
$$


for some constant $C_{2}$. Combining the above with (7), we have

$$
\int_{B_{p}(R)}|d *(\alpha \wedge \omega)|^{2} \leq C_{3} R^{-2} \int_{B_{p}(2 R)}|\alpha|^{2} .
$$

Let $R \rightarrow+\infty$, the result follows from the assumption that $\alpha$ is $L^{2}$ integrable.

Remark 6. Applications of the above theorem is available in the forthcoming paper [6].

\section{Holonomy and $\operatorname{Spin}(9)$ invariant}

We give a very brief introduction and list some basic principles about the holonomy group of a Riemannian manifold. We refer the readers to [2] and the references therein for further details. Most of the following introductory material are adopted from there. Let $p \in M$ and $\gamma:[0, l] \rightarrow M$ be a $C^{1}$-piecewise closed curve with $\gamma(0)=\gamma(l)=p$. Let $\tau(\gamma): T_{p} M \rightarrow T_{p} M$ be the parallel transport along $\gamma$. Since parallel transport preserves inner product, $\tau(\gamma)$ is an element of $O\left(T_{p} M\right)$, the orthogonal group of $T_{p} M$. Since the inverse of a curve $\gamma^{-1}$ and the composition of two curves $\gamma \cup \sigma$ satisfy $\tau\left(\gamma^{-1}\right)=(\tau(\gamma))^{-1}$ and $\tau(\gamma \cup \sigma)=\tau(\gamma) \circ \tau(\sigma)$. We can have the following definition:

Definition 7. The holonomy group (or the holonomy representation of $M$ at $p$ ) of a Riemannian manifold $(M, g)$ at $p$ is defined by

$$
\operatorname{Hol}(p)=\left\{\tau(\gamma): \gamma \in C^{1} \text {-piecewise closed curves of } M \text { based at } p\right\},
$$

the subgroup of the orthogonal group $O\left(T_{p} M\right)$.

On $M$, let us consider a tensor field $\alpha$. If $\alpha$ is invariant by parallel transport, that is, for any $p, q \in M$ and any curve $\gamma$ from $p$ to $q$, we have

$$
\tau^{*}(\gamma)(\alpha(p))=\alpha(q)
$$

where $\tau^{*}(\gamma)$ is the tensorial extension of the parallel transport $\tau(\gamma)$ along $\gamma$. By the above definition, $\alpha(p)$ at $T_{p} M$ is hence invariant by the tensorial extension of the holonomy representation $\operatorname{Hol}(p) \subseteq O\left(T_{p} M\right)$. Conversely, given any tensor on $T_{p} M$, if $\alpha_{0}$ is invariant under the tensorial extension of $\operatorname{Hol}(p)$, we can construct a tensor field $\alpha$ on $M$ by the formula $\tau^{*}(\gamma)(\alpha(p))=\alpha(q)$. Since $\alpha_{0}$ is invariant under the tensorial extension of $\operatorname{Hol}(p)$, the above definition is independent of the choice of the curve $\gamma$ and thus it is well-defined. Clearly, $\alpha(p)=\alpha_{0}$. By the above discussion, we have established a fundamental principle of holonomy group.

Proposition 8. Let $M$ be a Riemannian manifold and we consider a fixed type $(r, s)$ tensors on $M$. Then the following three properties are equivalent:

(1) There exists a tensor field of type $(r, s)$ which is invariant by parallel transport

(2) There exists $p \in M$ and a tensor $\alpha_{0}$ of type $(r, s)$ which is invariant by the tensorial extension of type $(r, s)$ of the holonomy representation $\operatorname{Hol}(p)$.

(3) There exists a tensor field $\alpha$ of type $(r, s)$ which has zero covariant derivative.

Proof. We have already established the equivalency of the first two statements in the discussion above. For the last statement, it can be seen easily via the formula

$$
(D \alpha)\left(X_{1}, \cdots, X_{s} ; X\right)=D_{X}\left(\alpha\left(X_{1}, \cdots, X_{s}\right)\right)-\sum_{i=1}^{s} \alpha\left(X_{1}, \cdots, D_{X} X_{i}, \cdots, X_{s}\right) .
$$


For any curve $\gamma$, let $X_{1}, \cdots, X_{s}$ be vector fields parallel along $\gamma$ and $X=\gamma^{\prime}$. Hence, the above equation becomes

$$
(D \alpha)\left(X_{1}, \cdots, X_{s} ; X\right)=D_{X}\left(\alpha\left(X_{1}, \cdots, X_{s}\right)\right) .
$$

Therefore, $D \alpha=0$ is equivalent to $D_{X}\left(\alpha\left(X_{1}, \cdots, X_{s}\right)\right)$, which implies $\alpha\left(X_{1}, \cdots, X_{s}\right)$ is constant along $\gamma$. Conversely, for any tangent vector $X(p)$, we can choose a curve $\gamma$ such that $\gamma^{\prime}=X(p)$.

Let $M$ be a manifold with holonomy group $\operatorname{Spin}(9)$. We are now ready to describe the parallel 8-form of $M$. The parallel 8-form of $\mathbb{H}_{\mathbb{N}}^{2}$ has been obtained by Brown and Gray in [3]. However, it is not easy to read off its properties for further applications because their 8-forms are defined via integration. In [1], the authors defined an 8form $\Omega$ and showed that it is $\operatorname{Spin}(9)$ invariant. In [13] the authors used the explicit formula of the parallel 4-form of a quaternionic Kähler manifold and proved that any harmonic function with finite Dirichlet integral is quaternionic-harmonic. Similarly, we will combine the explicit formula of $\Omega$ in [1] with a result in [13] to conclude that any harmonic function with finite Dirichlet integral is Cayley-harmonic. We now give a brief description of the $\operatorname{Spin}(9)$ invariant 8 -form $\Omega$ and we will follow the notations in [1]. For any point $p \in \mathbb{H}_{\mathbb{O}}^{2}$, we identify the tangent space at $p$ to the ordered pair of Cayley numbers, $T_{p}\left(\mathbb{H}_{\mathbb{O}}^{2}\right)=\mathbb{O}^{2}=\{(x, y): x, y \in \mathbb{O}\}$. Let $\bar{e}_{0}=1, \bar{e}_{1}, \cdots \bar{e}_{7}$ be a basis of $\mathbb{( 1 0}$ as in [15]. For any $x \in \mathbb{O}$, we let $x^{(2)}=(x, 0)$ and $x^{(3)}=(0, x)$. Let $\left\{v_{i}\right\}_{i=0}^{7}$ be the dual 1-forms of $\left\{\bar{e}_{i}^{(2)}\right\}_{i=0}^{7}$ and $\left\{w_{i}\right\}_{i=0}^{7}$ be the dual 1-forms of $\left\{\bar{e}_{i}^{(3)}\right\}_{i=0}^{7}$. Equivalently, we have

$$
\begin{aligned}
v_{i}\left(\bar{e}_{j}^{(2)}\right) & =\delta_{i j}, \quad v_{i}\left(\bar{e}_{j}^{(3)}\right)=0 \\
w_{i}\left(\bar{e}_{j}^{(2)}\right) & =0, \quad w_{i}\left(\bar{e}_{j}^{(3)}\right)=\delta_{i j},
\end{aligned}
$$

for any $0 \leq i, j \leq 7$. Let $e_{i}=\bar{e}_{i-1}^{(2)}$ for $1 \leq i \leq 8$ and $e_{j}=\bar{e}_{j-9}^{(3)}$ for $9 \leq j \leq 16$ so that $\left\{e_{i}\right\}_{i=1}^{16}$ is an orthonormal basis of $T_{p} \mathbb{H}_{\mathbb{O}}^{2}$.

$$
\omega_{i j}=v_{\sigma(i)} \wedge v_{\sigma(j)}, \quad \eta_{i j}=w_{\tau(i)} \wedge w_{\tau(j)},
$$

for some permutation functions $\sigma, \tau$ defined in [1]. For our purpose, we do not need to know the explicit forms of $\sigma, \tau$ and so we ignore it here for the sake of simplicity. Now we are ready to write down the formula of $\Omega$.

Theorem 9. [1] With the above notations,

$$
\Omega=\left(-v_{0} \wedge \cdots \wedge v_{7}+w_{0} \wedge \cdots \wedge w_{7}\right)+F\left(\omega_{i j}, \eta_{k l}\right)
$$

is Spin(9) invariant, where $F$ is a linear combinations of 8-forms, each of which is wedge products of some combinations of $\omega_{i j}, \eta_{k l}$.

We would like to point out that $F$ was given explicitly in [1]. However, the above simplified form of $\Omega$ is enough for our application.

Theorem 10. Let $M$ be a manifold with holonomy group Spin(9). Assume that $f$ is a harmonic function satisfying

$$
\int_{B_{p}(R)}|\nabla f|^{2}=o\left(R^{2}\right)
$$


as $R \rightarrow \infty$. Then with the above notations, we have

$$
\sum_{i=1}^{8} f_{i i}=0
$$

where $f_{i j}=\operatorname{Hess}(f)\left(e_{i}, e_{j}\right)$.

Proof. Fix $x \in M$ and let $\left\{e_{i}\right\}_{i=1}^{16}$ be the orthonormal frame of $T_{x} M$ in the above discussion. By the above construction, $\left\{\theta^{i}\right\}_{i=1}^{16}=\left\{v_{0}, \cdots, v_{7}, w_{0}, \cdots, w_{7}\right\}$ is the orthonormal coframe. By theorem 9,

$$
\Omega=\left(-v_{0} \wedge \cdots \wedge v_{7}+w_{0} \wedge \cdots \wedge w_{7}\right)+F\left(\omega_{i j}, \eta_{k l}\right)
$$

is $\operatorname{Spin}(9)$ invariant. Since $M$ has holonomy group $\operatorname{Spin}(9)$, proposition 8 implies that $\Omega$ can be extended to be a parallel form on $M$, which we still denote it by $\Omega$. By theorem 4, we have

$$
d *(d f \wedge \Omega)=0
$$

From (6), replacing $a_{i, j}$ by $f_{i j}$, the above equation is equivalent to

$$
\sum_{i, j=1}^{16} f_{i j} \varepsilon\left(\theta^{i}\right)\left(l\left(e_{j}\right) \Omega\right)=0 .
$$

Evaluate the above equation at $x$, we claim that the only terms containing $v_{0} \wedge \cdots \wedge v_{7}$ are the followings

$$
\sum_{i=1}^{8} f_{i i} \varepsilon\left(\theta^{i}\right)\left(l\left(e_{i}\right)\left(-v_{0} \wedge \cdots \wedge v_{7}\right)\right)=-\sum_{i=1}^{8} f_{i i} v_{0} \wedge \cdots \wedge v_{7}
$$

Since $d *(d f \wedge \Omega)=0$, we conclude that

$$
\sum_{i=1}^{8} f_{i i}=0
$$

at $x$. To prove the claim, since $l\left(e_{j}\right) F\left(\omega_{a b}, \eta_{c d}\right)$ kills off a $v_{j-1}$ term if $1 \leq j \leq 8$ or a $w_{j-9}$ term $9 \leq j \leq 16$ of $F\left(\omega_{a b}, \eta_{c d}\right)$. On the other hand, when $\varepsilon\left(\theta^{i}\right)$ acts on $l\left(e_{j}\right) F\left(\omega_{a b}, \eta_{c d}\right)$, it adds a $v_{i-1}$ term if $1 \leq i \leq 8$ or a $w_{i-9}$ term $9 \leq i \leq 16$ to $l\left(e_{j}\right) F\left(\omega_{a b}, \eta_{c d}\right)$. By the above discussion, since

$$
\omega_{a b}=v_{\sigma(a)} \wedge v_{\sigma(b)}
$$

and

$$
\eta_{a b}=w_{\tau(a)} \wedge w_{\tau(b)}
$$

it is easy to see that $\varepsilon\left(\theta^{i}\right)\left(l\left(e_{j}\right) F\left(\omega_{a b}, \eta_{c d}\right)\right)$ does not contain any terms of the form $v_{0} \wedge \cdots \wedge v_{7}$ and $w_{0} \wedge \cdots \wedge w_{7}$, for any $1 \leq i, j \leq 16$. This proved the claim and the result follows. 


\section{Manifolds with positive spectrum}

We summarize some useful properties of manifolds with positive spectrum. We refer the readers to [10] for a more detailed description on this subject. Let $M$ be a manifold with positive spectrum $\lambda_{1}(M)>0$. By the variational principle, it is equivalent to the following condition:

$$
\lambda_{1}(M) \int_{M} \phi^{2} \leq \int_{M}|\nabla \phi|^{2},
$$

for any compactly supported smooth function $\phi \in C_{c}^{\infty}(M)$. Since $\lambda_{1}(M)>0, M$ must be nonparabolic and it implies $M$ must have at least one nonparabolic end. $\lambda_{1}(M)>0$ also implies an end $E$ of $M$ is nonparabolic if and only if it has infinite volume. Assume that $M$ has at least two infinite volume ends, $E_{1}, E_{2}$. Let $B_{p}(R)$ be the geodesic ball with radius $R$ centered at $p$. We write $B(R)=B_{p}(R)$ when there is no ambiguity. We construct a sequence of harmonic functions $\left\{f_{R}\right\}$ by solving the following equation

$$
\left\{\begin{array}{rlc}
\triangle f_{R}=0 & \text { on } & B(R) \\
f_{R}=1 & \text { on } & \partial B(R) \cap E_{1} \\
f_{R}=0 & \text { on } & \partial B(R) \backslash E_{1}
\end{array}\right.
$$

By the theory of [8], $\left\{f_{R}\right\}$ converges (by passing to a subsequence if necessary) to a nonconstant harmonic function $f$ with finite Dirichlet integral on $M$ as $R \rightarrow+\infty$. Maximum principle implies that $0 \leq f \leq 1$. By the construction, it is clear that $\sup _{M} f=\sup _{E_{1}} f=1$ and $\inf _{M} f=\inf _{E_{2}} f=0$. We will need the following lemmas:

Lemma 11. [10] With the above notations, $f$ as constructed above. Then

(1)

$$
\begin{aligned}
\int_{E_{1}(R+1) \backslash E_{1}(R)}(1-f)^{2} & \leq C \exp \left(-2 \sqrt{\lambda_{1}(M)} R\right) \\
\int_{E(R+1) \backslash E(R)} f^{2} & \leq C \exp \left(-2 \sqrt{\lambda_{1}(M)} R\right)
\end{aligned}
$$

for some constant $C$ depends on $f, \lambda_{1}(M)$ and the dimension of $M$, where $E$ is any other end different from $E_{1}$.

(2)

$$
\int_{E(R+1) \backslash E(R)}|\nabla f|^{2} \leq C \exp \left(-2 \sqrt{\lambda_{1}(M)} R\right),
$$

for $R$ sufficiently large, where $E$ is any end of $M$.

Lemma 12. [12] For the function $f$ constructed above, let inf $f<a<b<\sup f$,

$$
l(t)=\{x \in M: f(x)=t\}
$$

and

Then

$$
\mathcal{L}(a, b)=\{x \in M: a<f(x)<b\} .
$$

$$
\int_{\mathcal{L}(a, b)}|\nabla f|^{2}=(b-a) \int_{l(b)}|\nabla f|
$$

and

$$
\int_{l(b)}|\nabla f|=\int_{l(t)}|\nabla f|
$$


for any $t \in(\inf f, \sup f)$.

\section{An one end result}

Theorem 13. Let $M$ be a complete noncompact 16-dimensional manifold with holonomy group Spin(9). Assume that the lowest spectrum satisfies $\lambda_{1}(M) \geq \frac{216}{7}$. Then $M$ has only one end with infinite volume.

Proof. Suppose that $M$ has at least two infinite volume ends, $E_{1}, E_{2}$. Since $\lambda_{1}(M)>$ $0, E_{1}, E_{2}$ must be nonparabolic. Let $f$ be the harmonic function constructed as in the previous section. Let $e_{1}=\frac{\nabla f}{|\nabla f|}$ and $\left\{e_{1}, \cdots, e_{8}, e_{9}, \cdots, e_{16}\right\}$ be a local orthonormal frame as in theorem 10 such that $e_{1} f=|\nabla f|, e_{\alpha} f=0,2 \leq \alpha \leq 16$ at a point $x$ and

$$
\sum_{i=1}^{8} f_{i i}=0
$$

hence we have

$$
\begin{aligned}
\sum_{i, j=1}^{16} f_{i j}^{2} & \geq f_{11}^{2}+\sum_{i=2}^{8} f_{i i}^{2}+2 \sum_{i=2}^{16} f_{1 j}^{2} \\
& \geq f_{11}^{2}+\frac{1}{7}\left(\sum_{i=2}^{8} f_{i i}\right)^{2}+2 \sum_{i=2}^{16} f_{1 j}^{2} \\
& \geq \frac{8}{7} \sum_{j=1}^{8} f_{1 j}^{2} \\
& =\left.\frac{8}{7}|\nabla| \nabla f\right|^{2}
\end{aligned}
$$

at $x$. Combining the above inequality with Bochner formula gives us

$$
\begin{aligned}
\frac{1}{2} \triangle|\nabla f|^{2} & =\sum_{i, j=1}^{16} f_{i j}^{2}+\operatorname{Ric}(\nabla f, \nabla f) \\
& \geq\left.\left.\frac{8}{7}|\nabla| \nabla f\right|^{2}\right|^{2}-36|\nabla f|^{2} .
\end{aligned}
$$

Let $g=|\nabla f|^{6 / 7}$, the above inequality becomes

$$
\triangle g \geq-\frac{216}{7} g .
$$

The variational principle of $\lambda_{1}(M)$ implies that for any compactly supported smooth function $\phi \in C_{c}^{\infty}(M)$, we have

$$
\begin{aligned}
\frac{216}{7} \int_{M} \phi^{2} g^{2} & \leq \int_{M}|\nabla(\phi g)|^{2} \\
& =\int_{M}\left(|\nabla \phi|^{2} g^{2}+|\nabla g|^{2} \phi^{2}+\frac{1}{2}\left\langle\nabla \phi^{2}, \nabla g^{2}\right\rangle\right) \\
& =\int_{M}|\nabla \phi|^{2} g^{2}-\int_{M} \phi^{2} g \triangle g .
\end{aligned}
$$


Combining the above with (8), we have

$$
\begin{aligned}
0 & \leq \int_{M} \phi^{2} g\left(\triangle g+\frac{216}{7} g\right) \\
& \leq \int_{M}\left|\nabla \phi^{2}\right| g^{2} .
\end{aligned}
$$

We choose $\phi=\psi \cdot \chi$ to be the product of two compactly smooth functions. For any $\varepsilon \in(0,1 / 2)$, we construct $\psi, \chi$ as follows

$$
\begin{gathered}
\chi(x)=\left\{\begin{array}{cll}
0 & \text { on } & \mathcal{L}(0, \varepsilon / 2) \cup \mathcal{L}(1-\varepsilon / 2,1) \\
(\log 2)^{-1}(\log f-\log (\varepsilon / 2)) & \text { on } & \mathcal{L}(\varepsilon / 2, \varepsilon) \cap\left(M \backslash E_{1}\right) \\
(\log 2)^{-1}(\log (1-f)-\log (\varepsilon / 2)) & \text { on } & \mathcal{L}(1-\varepsilon, 1-\varepsilon / 2) \cap E_{1} \\
1 & \text { otherwise }
\end{array} .\right. \\
\psi=\left\{\begin{array}{cll}
1 & \text { on } & B(R-1) \\
R-r & \text { on } & B(R) \backslash B(R-1) \\
0 & \text { on } & M \backslash B(R)
\end{array}\right.
\end{gathered}
$$

Then applying the right hand side of (9), we have

$$
\int_{M}|\nabla \phi|^{2} g^{2} \leq 2 \int_{M}|\nabla \psi|^{2} \chi^{2}|\nabla f|^{\frac{12}{7}}+2 \int_{M}|\nabla \chi|^{2} \psi^{2}|\nabla f|^{\frac{12}{7}} .
$$

$M$ is Einstein and the Ricci curvature satisfies $\operatorname{Ric}_{M}=-36$ under our normalization. The local gradient estimate of Cheng-Yau [4] (see also [11]) implies that

$$
|\nabla f| \leq C f
$$

for some constant $C$. The above inequality implies that

$$
|\nabla f| \leq C|1-f|,
$$

by replacing $f$ with $1-f$. On $E_{1}$, the first term of (10) can be estimated by

$$
\int_{E_{1}}|\nabla \psi|^{2} \chi^{2}|\nabla f|^{\frac{12}{7}} \leq\left(\int_{\Omega}|\nabla f|^{2}\right)^{6 / 7}\left(\int_{\Omega} 1\right)^{1 / 7}
$$

where $\Omega=E_{1} \cap(B(R) \backslash B(R-1)) \cap(\mathcal{L}(1-\varepsilon, 1-\varepsilon / 2) \cup \mathcal{L}(\varepsilon / 2, \varepsilon))$. Since $0<\varepsilon<1 / 2$, $\varepsilon / 2 \leq 1-f$ on $\Omega$ and we have

$$
\begin{aligned}
\int_{\Omega} 1 & \leq \int_{\Omega}\left(\frac{2(1-f)}{\varepsilon}\right)^{2} \\
& \leq C_{1} \varepsilon^{-2} \exp \left(-2 \sqrt{\lambda_{1}(M)} R\right)
\end{aligned}
$$

where the last inequality follows from lemma 11 . Combining lemma 11, the above inequality and (12), we conclude that

$$
\int_{E_{1}}|\nabla \psi|^{2} \chi^{2}|\nabla f|^{12 / 7} \leq C_{2} \varepsilon^{-2 / 7} \exp \left(-2 \sqrt{\lambda_{1}(M)} R\right) .
$$


The second term of (10) can be estimated by

$$
\begin{aligned}
\int_{E_{1}}|\nabla \chi|^{2} \psi^{2}|\nabla f|^{12 / 7} & \leq(\log 2)^{-2} \int_{\mathcal{L}(1-\varepsilon, 1-\varepsilon / 2) \cap E_{1} \cap B(R)}|\nabla f|^{12 / 7}|\nabla \log (1-f)|^{2} \\
& =(\log 2)^{-2} \int_{\mathcal{L}(1-\varepsilon, 1-\varepsilon / 2) \cap E_{1} \cap B(R)}|\nabla f|^{2+12 / 7}(1-f)^{-2} \\
& \leq C_{3} \int_{\mathcal{L}(1-\varepsilon, 1-\varepsilon / 2) \cap E_{1} \cap B(R)}|\nabla f|^{2}(1-f)^{-2 / 7},
\end{aligned}
$$

where the last inequality follows from (11). Co-area formula and lemma 12 give us

$$
\begin{aligned}
\int_{\mathcal{L}(1-\varepsilon, 1-\varepsilon / 2) \cap E_{1} \cap B(R)}|\nabla f|^{2}(1-f)^{-2 / 7} & =\int_{1-\varepsilon}^{1-\varepsilon / 2}(1-t)^{-2 / 7} \int_{l(t) \cap E_{1} \cap B(R)}|\nabla f| d A d t \\
& \leq \int_{l(b)}|\nabla f| d A \int_{1-\varepsilon}^{1-\varepsilon / 2}(1-t)^{-2 / 7} d t \\
& \leq C_{4} \varepsilon^{5 / 7} \int_{l(b)}|\nabla f| d A .
\end{aligned}
$$

Therefore, combing the above inequalities, (10) becomes

$$
\int_{E_{1}}|\nabla \phi|^{2} g^{2} \leq C_{5}\left(\varepsilon^{-2 / 7} \exp \left(-2 \sqrt{\lambda_{1}(M)} R\right)+\varepsilon^{5 / 7}\right) .
$$

Applying the same argument to $1-f$ instead of $f$ to the rest of the ends of $M$, we have

$$
\int_{M \backslash E_{1}}|\nabla \phi|^{2} g^{2} \leq C_{5}\left(\varepsilon^{-2 / 7} \exp \left(-2 \sqrt{\lambda_{1}(M)} R\right)+\varepsilon^{5 / 7}\right) .
$$

Combining (9), (14) and (15), letting $R \rightarrow+\infty$ and $\varepsilon \rightarrow 0$, we conclude that

$$
\triangle g=-\frac{216}{7} g
$$

and hence all the inequalities in proving (8) are indeed equalities. In particular, $\left(f_{\alpha \beta}\right)$ is diagonal and there exists a function $\mu$ such that

$$
\left(f_{\alpha \beta}\right)=\left(\begin{array}{ccc}
-7 \mu & & \\
& D_{1} & \\
& & D_{2}
\end{array}\right),
$$

where $D_{1}=\mu I$ and $D_{2}$ is the $8 \times 8$ zero matrix. Since $f_{1 \alpha}=0$ for any $\alpha \neq 1$, $|\nabla f|$ is constant along the level set of $f$. In particular, the level sets of $|\nabla f|$ and $f$ coincide. Suppose $|\nabla f|(x)=0$, by considering $f+c$, we may assume that $f(x)=0$. The regularity theory of harmonic functions asserts that $f$ locally in a neighborhood of $x$ behaves like a homogeneous harmonic polynomial in $\mathbb{R}^{n}$ with the origin at $x$. This is impossible since the level sets of $|\nabla f|$ and $f$ coincide. Hence $|\nabla f| \neq 0$ on $M$ and $M$ is diffeomorphic to $\mathbb{R} \times N$, where $N$ is given by the level set of $f$. $N$ is compact since we have assumed that $M$ has at least two ends. Fix a level set $N$ of $f$. We choose a local orthonormal frame $\left\{e_{i}\right\}_{\alpha=2}^{16}$ of $N$ and $e_{1}=\frac{\nabla f}{|\nabla f|}$. Let $\gamma(t)$ be the integral curve of $e_{1}$ and $\left\{e_{\alpha}(t)\right\}_{\alpha=2}^{16}$ be the parallel transport of $\left\{e_{\alpha}\right\}_{\alpha=2}^{16}$ along $\gamma$. $\left\langle\nabla_{e_{1}} e_{1}, e_{1}\right\rangle=0=\left\langle\nabla_{e_{1}} e_{1}, e_{\alpha}\right\rangle$ for any $\alpha \geq 2$ implies

$$
\nabla_{e_{1}} e_{1}=0
$$


and hence $\gamma$ is a geodesic. The second fundamental form of the level set of $f$ satisfies the following equations

$$
\begin{aligned}
f_{\alpha \beta} & =e_{\alpha} e_{\beta} f-\left(\nabla_{e_{\alpha}} e_{\beta}\right) f \\
& =\left\langle-\left(\nabla_{e_{\alpha}} e_{\beta}\right), e_{1}\right\rangle f_{1} \\
& =h_{\alpha \beta} f_{1} \\
\nabla_{e_{\alpha}} e_{1} & =\sum_{\beta=2}^{16} h_{\alpha \beta} e_{\beta}
\end{aligned}
$$

where $h_{\alpha \beta}=\left\langle-\left(\nabla_{e_{\alpha}} e_{\beta}\right), e_{1}\right\rangle$ is the second fundamental form of $N$. We now compute the curvature of $M$

$$
\begin{aligned}
\left\langle R\left(e_{1}, e_{\alpha}\right) e_{1}, e_{\alpha}\right\rangle & =\left\langle\nabla_{e_{1}} \nabla_{e_{\alpha}} e_{1}-\nabla_{e_{\alpha}} \nabla_{e_{1}} e_{1}-\nabla_{\left[e_{1}, e_{\alpha}\right]} e_{1}, e_{\alpha}\right\rangle \\
& =\left\langle\nabla_{e_{1}} \nabla_{e_{\alpha}} e_{1}, e_{\alpha}\right\rangle-\left\langle\nabla_{\left[e_{1}, e_{\alpha}\right]} e_{1}, e_{\alpha}\right\rangle \\
& =\left\langle\nabla_{e_{1}} \nabla_{e_{\alpha}} e_{1}, e_{\alpha}\right\rangle-\left\langle\nabla_{\left.\nabla_{e_{1}} e_{\alpha}-\nabla_{e_{\alpha}} e_{1}, e_{\alpha}\right\rangle}\right. \\
& =\left\langle\nabla_{e_{1}} \nabla_{e_{\alpha}} e_{1}, e_{\alpha}\right\rangle+\sum_{\beta=2}^{16}\left\langle\nabla_{e_{\alpha}} e_{1}, e_{\beta}\right\rangle\left\langle\nabla_{e_{\beta}} e_{1}, e_{\alpha}\right\rangle \\
& =\sum_{\beta=2}^{16}\left\langle e_{1}\left(h_{\alpha \beta}\right) e_{\beta}, e_{\alpha}\right\rangle+\sum_{\beta=2}^{16} h_{\alpha \beta}^{2} \\
& =e_{1}\left(h_{\alpha \alpha}\right)+h_{\alpha \alpha}^{2},
\end{aligned}
$$

where we have used $(17),(18)$ and the fact that $\left(f_{\alpha \beta}\right)$ is diagonal. In particular, since $M$ is covered by $\mathbb{H}_{\mathbb{O}}^{2},(16)$ and (19) implies the sectional curvature

$$
K_{M}\left(e_{1}, e_{k}\right)=K_{\mathbb{H}_{\mathbb{\Phi}}^{2}}\left(e_{1}, e_{k}\right)=0,
$$

for any $k \geq 9$. It contradicts to the fact that the sectional curvature of $\mathbb{H}_{\mathbb{O}}^{2}$ is pinched between -4 and -1 . Therefore, $M$ has only one infinite volume end.

\section{Splitting type theorem}

Theorem 14. Let $M$ be a complete noncompact 16-dimensional manifold with holonomy group Spin(9). Assume that the lowest spectrum of $M$ achieves the maximal value, that is $\lambda_{1}(M)=121$. Then either

(1) $M$ has only one end; or

(2) $M$ is diffeomorphic to $\mathbb{R} \times N$ with metric

$$
d s_{M}^{2}=d t^{2}+e^{-4 t} \sum_{k=2}^{8} \omega_{k}^{2}+e^{-2 t} \sum_{k=9}^{16} \omega_{k}^{2},
$$

where $\left\{\omega_{2}, \cdots, \omega_{16}\right\}$ is an orthonormal basis for a compact manifold $N$ given by a compact quotient of the horosphere of the universal cover $\tilde{M}$ of $M$.

Proof. Since $\lambda_{1}(M)>0, M$ is nonparabolic and hence $M$ has at least one nonparabolic end. Assume that $M$ has at least two ends. Theorem 13 implies that $M$ must have a parabolic end. Let $E_{1}$ be a nonparabolic end and $E_{2}$ be a parabolic end with respect to $B_{p}\left(R_{0}\right)$, the geodesic ball with radius $R_{0}$ centered at $p$. In other words, $E_{1}, E_{2}$ are two unbounded component of $M \backslash B_{p}\left(R_{0}\right)$. Let $\bar{\gamma}:[0,+\infty) \rightarrow M$ 
be a geodesic ray with $\bar{\gamma}(0)=p$ and $\bar{\gamma}\left(\left[R_{0},+\infty\right)\right) \subseteq E_{2}$, for some $a>0$. Let $\beta(x)=\lim _{t \rightarrow \infty}(t-r(x, \bar{\gamma}(t)))$ be the Busemann function with respect to $\bar{\gamma}$. Theorem 2 gives us

$$
\triangle r(x, \bar{\gamma}(t)) \leq 14 \operatorname{coth}(2 r(x, \bar{\gamma}(t)))+8 \operatorname{coth} r(x, \bar{\gamma}(t)),
$$

which implies

$$
\triangle \beta \geq-22,
$$

in the sense of distribution. Let $f=\exp (11 \beta)$, we compute

$$
\begin{aligned}
\Delta f & =11 f \triangle \beta+121 f|\nabla \beta|^{2} \\
& \geq-121 f .
\end{aligned}
$$

Using the variation principle of $\lambda_{1}(M)=121$, for any $\phi \in C_{c}^{\infty}(M)$ nonnegative smooth function with compact support, we have

$$
\begin{aligned}
121 \int_{M} \phi^{2} f^{2} & \leq \int_{M}|\nabla(\phi f)|^{2} \\
& =\int_{M}|\nabla \phi|^{2} f^{2}+\frac{1}{2} \int_{M}\left\langle\nabla \phi^{2}, \nabla f^{2}\right\rangle+\int_{M} \phi^{2}|\nabla f|^{2} \\
& =\int_{M}|\nabla \phi|^{2} f^{2}-\int_{M} \phi^{2} f \triangle f,
\end{aligned}
$$

thus

$$
\int_{M} \phi^{2} f(\triangle f+121 f) \leq \int_{M}|\nabla \phi|^{2} f^{2} .
$$

Follow the argument in [9], if we choose the following cut-off function

$$
\phi(x)=\left\{\begin{array}{ccl}
1 & \text { on } & B_{p}(R) \\
\frac{2 R-r(x)}{R} & \text { on } & B_{p}(2 R) \backslash B_{p}(R) \\
0 & \text { on } & M \backslash B_{p}(R)
\end{array},\right.
$$

then the right hand side of (20) converges to zero as $R \rightarrow+\infty$. Indeed,

$$
\int_{M}\left|\nabla \phi^{2}\right| f^{2} \leq R^{-2} \int_{\left(B_{p}(2 R) \backslash B_{p}(R)\right) \cap E_{2}} f^{2}+R^{-2} \int_{\left(B_{p}(2 R) \backslash B_{p}(R)\right) \backslash E_{2}} f^{2} .
$$

For an end $E$, let $V_{R}(E)$ be the volume of the set $B_{p}(R) \cap E$ and let $k-1 \leq R<k$. The first term on the right hand side of (21) can be estimated by

$$
\begin{aligned}
\int_{\left(B_{p}(2 R) \backslash B_{p}(R)\right) \cap E_{2}} f^{2} & \leq \sum_{i=1}^{k} \int_{\left(B_{p}(R+i) \backslash B_{p}(R+i-1)\right) \cap E_{2}} f^{2} \\
& \leq \sum_{i=1}^{k} e^{22(R+i)}\left(V_{E_{2}}(R+i) \backslash V_{E_{2}}(R+i-1)\right) \\
& \leq C_{1} \sum_{i=1}^{k} e^{22(R+i)} e^{-22(R+i-1)} \\
& \leq C_{2} R
\end{aligned}
$$


where the second inequality follows from $|\beta(x)| \leq r(x, p)$ and the third inequality follows from the volume estimate on a parabolic end $E$ of [10],

$$
V_{\infty}(E)-V_{R}(E) \leq C \exp (-2 \sqrt{\lambda(E)} R) \text { if } \lambda(E)>0 .
$$

On the other hand, let $\tau$ be the geodesic ray given in lemma 3. For any $x \in M \backslash$ $\left(B_{p}\left(R_{0}\right) \cup E_{2}\right)$, then $\tau$ must intersect $B_{p}\left(R_{0}\right)$. Let $y$ to be the first point on $\tau$ that intersects $B_{p}\left(R_{0}\right)$, it is easy to see that $([9])$

$$
\begin{aligned}
\beta(y)-\beta(x) & \geq r(x, y) \\
& \geq r(x, p)-r(y, p),
\end{aligned}
$$

and hence

$$
\begin{aligned}
\beta(x) & \leq-r(x, p)+r(y, p)+\beta(y) \\
& \leq-r(x, p)+2 r(y, p) \\
& \leq-r(x, p)+2 R_{0},
\end{aligned}
$$

and hence the second term of the right hand side of (21) can now be estimated by

$$
\begin{aligned}
(23) \int_{\left(B_{p}(2 R) \backslash B_{p}(R)\right) \backslash E_{2}} f^{2} & \leq \sum_{i=1}^{k} \int_{\left(B_{p}(R+i) \backslash B_{p}(R+i-1)\right) \backslash E_{2}} f^{2} \exp \left(44 R_{0}-22 r(x, p)\right) \\
& \leq \sum_{i=1}^{k} \int_{\left(B_{p}(R+i) \backslash B_{p}(R+i-1)\right) \backslash E_{2}} \\
& \leq \sum_{i=1}^{k} C_{3} e^{-22(R+i-1)} V\left(B_{p}\left(R_{0}+i\right)\right) \\
& \leq \sum_{i=1}^{k} C_{3} e^{-22(R+i-1)} e^{22(R+i)} \\
& \leq C_{4} R .
\end{aligned}
$$

Combining (21), (22) and (23), we conclude that the right hand side of (20) converges to zero as $R \rightarrow+\infty$. Since $f$ is non-negative, (20) now implies

$$
\triangle f+121 f=0,
$$

and all inequalities in the proving (20) are indeed equalities and in particular,

$$
\triangle \beta=-22, \quad|\nabla \beta|=1,
$$

and $\beta$ is smooth by the regularity of the above equation. The above equation implies $M$ is diffeomorphic to $\mathbb{R} \times N$, where $N$ is diffeomorphic to the level set of $\beta$. $N$ is compact since otherwise $M$ would have only one end, contradicts to our assumption that $M$ has two ends. Let $N_{0}$ be the level set of $\beta$ with $x \in N_{0}$. Let $e_{1}=\nabla \beta(x)=\tau^{\prime}(x)$ be the unit normal direction of $N_{0}$ at $x$, where $\tau$ was the geodesic ray given in lemma 3. Let $\gamma(t)$ be the integral curve of $\nabla \beta$ with $\gamma(0)=x \in N_{0}$ and $e_{1}(t)=\gamma^{\prime}(t)$. We pick a local orthonormal frame $\left\{e_{i}\right\}_{A=2}^{16}$ of $N_{0}$ around $x$ as in the proof of proposition 1 such that

$$
\begin{aligned}
R_{1 i 1 i}(x) & =-4,2 \leq i \leq 8 \\
R_{1 \alpha 1 \alpha}(x) & =-1,9 \leq \alpha \leq 16
\end{aligned}
$$


at $x$. We extend the frame to a local orthonormal frame $\left\{e_{A}(t)\right\}_{A=2}^{16}$ along $\gamma$ by parallel transport. $e_{1}\left\langle e_{1}, e_{\alpha}\right\rangle=0=e_{1}\left\langle e_{1}, e_{1}\right\rangle$ implies $\nabla_{e_{1}} e_{1}=0$, thus $\gamma(t)$ is a normal geodesic with $\gamma^{\prime}(0)=\tau^{\prime}(x)$. Therefore $\gamma \equiv \tau$. As in the proof of proposition 1, we have

$$
\begin{aligned}
R_{1 i 1 i}(\gamma(t)) & =-4,2 \leq i \leq 8 \\
R_{1 \alpha 1 \alpha}(\gamma(t)) & =-1,9 \leq \alpha \leq 16
\end{aligned}
$$

along $\gamma$. Bochner formula gives us

$$
\begin{aligned}
0 & =\frac{1}{2} \triangle|\nabla \beta|^{2} \\
& =\sum_{i, j=1}^{16} \beta_{i j}^{2}+\operatorname{Ric}(\nabla \beta, \nabla \beta)+\langle\nabla \beta, \nabla \triangle \beta\rangle \\
& =\sum_{i, j=1}^{16} \beta_{i j}^{2}-36
\end{aligned}
$$

The proof of proposition 1 with $e_{1}=\gamma^{\prime}(0)=\tau^{\prime}(x)$ implies

$$
\beta_{11}=0, \quad \sum_{i=2}^{8} \beta_{i i} \geq-14, \quad \sum_{\alpha=9}^{16} \beta_{\alpha \alpha} \geq-8,
$$

where the first equality comes from the fact that $\beta$ is linear along $\tau$ (lemma 3). Combining the above with (24) implies

$$
\sum_{i=2}^{8} \beta_{i i}=-14, \sum_{\alpha=9}^{16} \beta_{\alpha \alpha}=-8 .
$$

Combining (25) and (26), we have

$$
\begin{aligned}
36 & =\sum_{A, B=1}^{16} \beta_{A B}^{2} \\
& \geq \sum_{i=2}^{8} \beta_{i i}^{2}+\sum_{\alpha=9}^{16} \beta_{\alpha \alpha}^{2} \\
& \geq \frac{1}{7}\left(\sum_{i=2}^{8} \beta_{i i}\right)^{2}+\frac{1}{8}\left(\sum_{\alpha=9}^{16} \beta_{\alpha \alpha}\right)^{2} \\
& =36 .
\end{aligned}
$$

Therefore all inequalities in the above proof are indeed equalities $\beta_{A B}$ is diagonal and

$$
\beta_{A B}=-c_{A} \delta_{A B}
$$

where

$$
c_{A}=\left\{\begin{array}{cc}
0 & A=1 \\
2 & 2 \leq A \leq 8 \\
1 & 9 \leq A \leq 16
\end{array}\right.
$$


The second fundamental form of the each level set $N_{t}=\{x \in M: \beta(x)=t\}$ with respect to the normal vector $\nabla \beta$ can now be calculated

$$
\begin{aligned}
h_{\sigma \tau} & =\left\langle-\nabla_{e_{\sigma}} e_{\tau}, e_{1}\right\rangle \\
& =\left\langle-\nabla_{e_{\sigma}} e_{\tau}, \nabla \beta\right\rangle \\
& =-\left(\nabla_{e_{\sigma}} e_{\tau}\right) \beta \\
& =\beta_{\sigma \tau},
\end{aligned}
$$

where $2 \leq \sigma, \tau \leq 16$ and the last equality follows from the fact that $N_{t}$ is a level set of $\beta$. In particular, we have

$$
\nabla_{e_{\sigma}} e_{1}=\sum_{\tau=2}^{16} \beta_{\sigma \tau} e_{\tau} .
$$

For any $p \in N_{0}$, let $\gamma(t)$ be the integral curve of $\nabla \beta$ with $\gamma(0)=p$. Define $\bar{\psi}_{t}(p)=\gamma(t)$, and it induces a map $\psi_{t}: N_{0} \rightarrow N_{t}$. As we have already seen that the integral curve of $\nabla \beta$ is a normal geodesic, $\sigma(t)=\psi_{t}(\cdot)$ is always a normal geodesic and thus $\psi_{t}$ is a geodesic flow on $M$, therefore $d \psi_{t}(X)$ is a Jacobi field along each integral curve. Let $\bar{e}_{k}$ be the restriction of $e_{k}$ on $N_{0}, \quad 1 \leq k \leq 16$. We claim that $d \psi_{t}\left(\bar{e}_{i}\right)=V_{i}(t)$, where

$$
V_{A}(t)=e^{-c_{A} t} e_{A}(t), \quad 2 \leq A \leq 16 .
$$

By the uniqueness of Jacobi field, it is sufficient to show that $V_{A}(t)$ satisfies the Jacobi equation with the same initial conditions as $d \psi_{t}\left(\bar{e}_{A}\right)$. We have

$$
\begin{aligned}
\nabla_{\gamma^{\prime}} \nabla_{\gamma^{\prime}} V_{A} & =-c_{A}^{2} e_{A} \\
& =R_{1 A 1 A} e_{A} \\
& =R\left(\gamma^{\prime}, V\right) \gamma^{\prime},
\end{aligned}
$$

since $R_{A B}=R_{1 A 1 B}$ is diagonal. On the other hand, $V_{A}(0)=\bar{e}_{A}=d \psi_{0}\left(\bar{e}_{A}\right)$ and (28) implies

$$
\begin{aligned}
\nabla_{\gamma^{\prime}}\left(d \psi_{t}\left(\bar{e}_{A}\right)\right)(0) & =\nabla_{\bar{e}_{A}} e_{1}(0) \\
& =\sum_{\tau=2}^{16} \beta_{A \tau} \bar{e}_{\tau} \\
& =-c_{A} \bar{e}_{A},
\end{aligned}
$$

since we can view $e_{1}$ and $d \psi_{t}\left(\bar{e}_{A}\right)$ as tangent vectors of a map from a rectangle. Therefore $V_{A}^{\prime}(0)=-c_{A} \bar{e}_{A}=\nabla_{\gamma^{\prime}}\left(d \psi_{t}\left(\bar{e}_{A}\right)\right)(0)$. In conclusion, each $N_{t}$ can be viewed as a copy of $N_{0}$ and $M$ is diffeomorphic $\mathbb{R} \times N_{0}$ with the metric

$$
d s_{M}^{2}=d t^{2}+e^{-4 t} \sum_{k=2}^{8} \omega_{k}^{2}+e^{-2 t} \sum_{k=9}^{16} \omega_{k}^{2},
$$

where $\left\{\omega_{k}\right\}_{k=2}^{16}$ is the coframe of $\left\{\bar{e}_{2}, \cdots, \bar{e}_{16}\right\}$. 


\section{Acknowledgements}

The author would like to express his deepest gratitude to his advisor Prof. Peter Li for his continuous support and guidance, without which the author would not be able to complete this work. The author would also like to thank Prof. Jiaping Wang and Prof. Roger Chen for their interests in this subject.

\section{References}

[1] K. Abe and M. Matsubara, Invariant forms on the exceptional symmetric spaces FII and EIII, Korea Adv. Inst. Sci. Tech., Taejŏn (1996) 3-16.

[2] A. Besse, Einstein manifolds, Springer-Verlag, Berlin (1987).

[3] R. Brown and A. Gray, Riemannian manifolds with holonomy group Spin(9), Diff. Geometry in honor of K. Yano, Kinokuniya, Tokyo (1972) 41-59.

[4] S. Y. Cheng and S. T. Yau, Differential equations on Riemannian manifolds and their geometric applications, Comm. Pure App. Math. 28 (1975) 333-354.

[5] K. Corlette, Hausdorff dimensions of limit sets, Invent. Math 102 (1990) 521-541.

[6] K. H. Lam, Results on weighted Poincare inequality of complete manifolds, to appear in Transactions of the American Mathematical Society.

[7] P. Li, On the structure of complex Kahler manifolds with nonnegative curvature near infinity, Invent. Math. 99 (1990) 579-600.

[8] P. Li and L. F. Tam, Harmonic functions and the structure of complete manifolds, J. Diff. Geom. 35 (1992) 359-383.

[9] P. Li and J. Wang, Connectedness at infinity of complete Kahler manifolds, Preprint.

[10] - Complete manifolds with positive spectrum, J. Diff. Geom. 58 (2001) 501-534.

[11] — Complete manifolds with positive spectrum II, J. Diff. Geom. 62 (2002) 143-162.

[12] —, Weighted Poincaré inequality and rigidity of complete manifolds, Ann. Scient. Éc. Norm. Sup., 4e série, t. 39 (2006) 921-982.

[13] S. Kong, P. Li, and D. Zhou, Spectrum of the Laplacian on quaternionic Kahler manifolds, J. Diff. Geom. 78 (2008), 295-332.

[14] Y. T. Siu, The complex-analyticity of harmonic maps and the strong rigidity of compact Kähler manifolds, Ann. Math. 112 (1980) 73-111.

[15] I. Yokota, Exceptional Lie group $F_{4}$ and its representation rings, J. Fac. Sci. Shinshu Univ. (1968) 35-60.

Department of mathematics, National Cheng Kung University, National Center for Theoretical Sciences, Tainan, Taiwan 70101

E-mail address: khlam@mail.ncku.edu.tw 